\title{
Contagion Flow through Banking Networks
}

\author{
Michael Boss ${ }^{1}$, Martin Summer ${ }^{1}$, and Stefan Thurner ${ }^{2}$ \\ 1 Oesterreichische Nationalbank, Otto-Wagner-Platz 3, A-1011 Wien, Austria* \\ 2 Complex Systems Research Group, HNO, Medical University of Vienna, \\ Währinger Gürtel 18-20, A-1090 Vienna, Austria \\ thurner@univie.ac.at
}

\begin{abstract}
Starting from an empirical analysis of the network structure of the Austrian inter-bank market, we study the flow of funds through the banking network following exogenous shocks to the system. These shocks are implemented by stochastic changes in variables like interest rates, exchange rates, etc. We demonstrate that the system is relatively stable in the sence that defaults of individual banks are unlikely to spread over the entire network. We study the contagion impact of all individual banks, meaning the number of banks which are driven into insolvency as a result of a single bank's default. We show that the vertex betweenness of individual banks is linearly related to their contagion impact.
\end{abstract}

\section{Introduction}

With the development of new ideas and tools of analysis, physics has lately strongly contributed to a functional understanding of the structure of complex real world networks. A key insight of this research has been the discovery of surprising structural similarities in seemingly very different networks, ranging from internet technology to cell biology. See 11 for an overview. Remarkably, many real world networks show power-law (scale free) degree distributions and feature a certain pattern of cliquishness, quantified by the clustering coefficient. Many also exhibit a so called small world phenomenon, meaning that the average shortest path between any two vertices ("degrees of separation") in the network can be surprisingly small. Maybe one of the most important contributions to recent network theory is an interpretation of these network parameters with respect to stability, robustness, and efficiency of an underlying system. From this perspective financial networks are a natural candidate to study. The financial world can be thought of as a set of intermediaries i.e. banks who interact with each other through financial transactions. These interactions are governed by a set of rules and regulations, and take place on an interaction graph of all connections between financial intermediaries. Financial crises i.e. the incapacity to finance businesses and industries have recently hit several countries all around the globe. These events have triggered a boom of papers on banking-crises, financial risk-analysis and numerous policy initiatives to find and understand the

\footnotetext{
* The views and findings of this paper are entirely those of the authors and do not necessarily represent the views of Oesterreichische Nationalbank.
} 
weak-points of the financial system. One of the major concerns in these debates is the danger of so called systemic risk: the large scale breakdown of financial intermediation due to domino effects of insolvency [23]. The network of mutual credit relations between financial institutions is supposed to play a key role in the risk for contagious defaults.

In the past the theoretical economic literature on contagion [4.5] suggest network topologies that might be interesting to look at. In 4 ] it is suggested to study a complete graph of mutual liabilities. The properties of a banking system with this structure is then compared to properties of systems with non complete networks. In [5] a set of different network structures is studied. However, so far surprisingly little is known about the actual empirical network topology of mutual credit relations between financial institutions.

In a recent paper we have for the first time analyzed empirical data to reconstruct the banking network of Austria [6]. Here we characterized the interbank network by the liability (or exposure) matrix $L$. The entries $L_{i j}$ are the liabilities bank $i$ has towards bank $j$. $L$ is a square matrix but not necessarily symmetric. We showed that the liability $\left(L_{i j}\right)$ size distribution follows a power law, which can be understood as being driven by underlying size and wealth distributions of the banks, which show similar power exponents. We find that the interbank network shows - like many other realistic networks - power law dependencies in the degree distributions. We could show that different scaling exponents within the same distribution relate to different hierarchy levels in sub-networks (sectors) within the total network. The scaling exponents by the agricultural banks are very low, due to the hierarchical structure of this sector, while the other banks lead to scaling exponents of sizes also found in other complex real world networks. The interbank network shows a low clustering coefficient, a result that mirrors the analysis of community structure which shows a clear network pattern, where banks would first have links with their head institution, whereas these few head institutions hold links among each other. A consequence of this structure is that the interbank network is a small world with a very low "degree of separation" between any two nodes in the system.

\section{Inter-bank Topology and Flow of Contagion}

The knowledge of the detailed structure of the interbank topology enables us to use it as an input for a contagion-flow model [7, which is the main idea of this paper. Unlike previous research where we studied random flow on structured networks 89, here we follow the flow of payments uniquely determined by the structure of the liability matrix. We use this flow to perform stress tests to the system by artificially changing external financial parameters like interest rates, exchange rates etc., mimicking conditions and "global" events which are beyond the influence of banks. By doing this we can answer questions about the stability of the financial system with respect to external shocks, in particular, which banks are likely to default due to shocks, and which banks will drag other banks into default due to their mutual credit relations (contagion). 
In the following we are looking for the bilateral liability matrix $L$ of all (about $N=900)$ Austrian banks, the Central Bank (OeNB) and an aggregated foreign banking sector. Our data consists of $10 \mathrm{~L}$ matrices each representing liabilities for quarterly single month periods between 2000 and 2003. T obtain these data, we draw upon two major sources from the Austrian Central Bank: the Austrian bank balance sheet data base (MAUS) and the major loan register (GKE). The Austrian banking system has a sectoral organization due to historic reasons. Banks belong to one of seven sectors: savings banks (S), Raiffeisen (agricultural) banks (R), Volksbanken (VB), joint stock banks (JS), state mortgage banks (SM), housing construction savings and loan associations (HCL), and special purpose banks (SP). Banks have to break down their balance sheet reports on claims and liabilities with other banks according to the different banking sectors, Central Bank and foreign banks. This practice of reporting on balance interbank positions breaks the liability matrix $L$ down to blocks of sub-matrices for the individual sectors. Banks with a head institution have to disclose their positions with the head institution, which gives additional information on $L$. Since many banks in the system hold interbank liabilities only with their head institutions, one can pin down many entries in the $L$ matrix exactly. This information is combined with the data from the major loans register of OeNB. This register contains all interbank loans above a threshold of 360000 Euro. This information provides us with a set of constraints and zero restrictions for individual entries $L_{i j}$. Up to this point one can obtain about $90 \%$ of the $L$-matrix entries exactly. For the rest we employ entropy maximization method [6]. The estimation problem can be set up as a standard convex optimization problem: Assume we have a total of $K$ constraints. The column and row constraints take the form $\sum_{j=1}^{N} L_{i j}=b_{i}^{r} \quad \forall \quad i$ and $\sum_{i=1}^{N} L_{i j}=b_{j}^{c} \quad \forall \quad j$ with $r$ denoting row and $c$ denoting column. Constraints imposed by the knowledge about particular entries in $L_{i j}$ are given by $b^{l} \leq L_{i j} \leq b^{u}$ for some $i, j$ The aim is to find the matrix $L$ that has the least discrepancy to some a priori matrix $U$ with respect to the (generalized) cross entropy measure

$$
\mathcal{C}(L, U)=\sum_{i=1}^{N} \sum_{j=1}^{N} L_{i j} \ln \left(\frac{L_{i j}}{U_{i j}}\right)
$$

$U$ is the matrix which contains all known exact liability entries. For those entries (bank pairs) ij where we have no knowledge from Central Bank data, we set $U_{i j}=1$. We use the convention that $L_{i j}=0$ whenever $U_{i j}=0$ and define $0 \ln \left(\frac{0}{0}\right)$ to be 0 . As a result we obtain a rather precise picture of the interbank relations at a particular point in time. Given $L$ we find that the distribution of liabilities follows a power law for more than three decades with an exponent of -1.87 [ 6 . To extract the network topology from these data, for the present purposes we ignore directions and define an undirected but weighted adjacency matrix $A_{i j}^{w}=L_{i j}+L_{j i}$, which measures the gross interbank interaction, i.e. the total volume of liabilities and assets for each node. We next test the validity of our estimate of $L$, by computing the implied community structure and then comparing it to the known real bank-clusters, i.e. the sectors. There exist various 


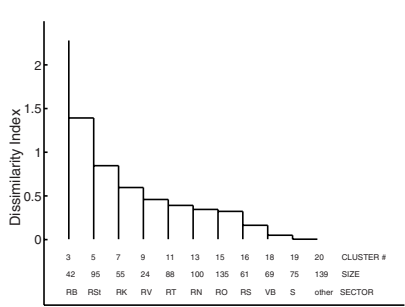

(a)

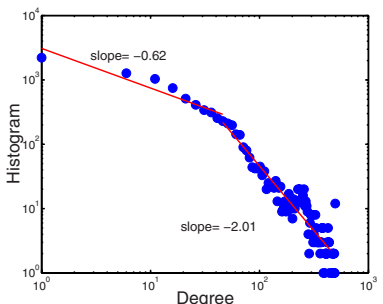

(b)

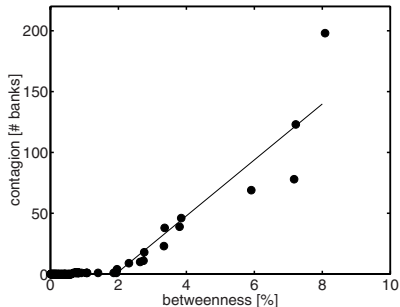

(c)

Fig. 1. (a) Community structure of the Austrian interbank market network from the same data $\left(A_{\text {clip }}^{w}\right)$. The dissimilarity index is a measure of the "differentness" of the clusters. (b) Empirical degree distribution of the interbank connection network. Histograms are from aggregated data from the 10 datasets. (c) Contagion impact as a function of relative node betweenness for the $L$ matrix. Below a value of $B(i)$ of 0.6 no contagion impact is found.

ways to find functional clusters within a given network. In [10 an algorithm was introduced which - while having at least the same performance rates as [11] - provides an additional measure for the differences of cluster, the so-called dissimilarity index. For analyzing our interbank network we apply this latter algorithm to the weighted adjacency matrix $A^{w}$. As the only preprocessing step we clip all entries in $A^{w}$ above a level of $300 \mathrm{~m}$ Euro for numerical reasons, i.e. $A_{\text {clip }}^{w}=\min \left(A^{w}, 300 \mathrm{~m}\right)$. The community structure obtained in this way can be compared to the actual community structure in the real world. The result for the community structure obtained from one representative data set is shown in Fig. 11a. The algorithm associates banks with their corresponding sectors, like R, $\mathrm{VB}$, and S. For banks which in reality are not structured in a strong hierarchical way, such as banks in the SP, JS, SM, HCL sectors, no significant community structure is expected. By the algorithm these banks are grouped together in a cluster called 'other'. The Raiffeisen sector, which has a sub-structure in the federal states, is further grouped into clusters which are clearly identified as $\mathrm{R}$ banks within one of the eight federal states (B,St,K,V,T,N,O,S). In Fig. 1 a these clusters are marked as e.g. 'RS', 'R' indicating the Raiffeisen sector, and 'S' the state of Salzburg. Overall, there were 31 mis-specifications into wrong clusters within the total $N=883$ banks, which is a mis-specification rate of $3.5 \%$, demonstrating the quality of the dissimilarity algorithm and - more importantly - the quality of the entropy approach to reconstruct the matrix $L$.

Like many real world networks, the degree distribution of the interbank market follows a power law for the tail of graph $A$, Fig. 1 b. The exponent is $\gamma_{\text {tail }}(A)=2.01$. We have checked that the distribution for the low degrees is almost entirely dominated by banks of the $\mathrm{R}$ sector. Typically in the $\mathrm{R}$ sector most small agricultural banks have links to their federal state head institution and very few contacts with other banks, leading to a strong hierarchical structure. This hierarchical structure is perfectly reflected by the small scaling exponents. Betweenness is a measure of centrality that considers the position of nodes in-between the shortest paths (geodesics) that link any other nodes of the net- 
work. Let $g_{j k}$ be the number of geodesics linking nodes $j$ and $k$. If all geodesics are equally likely to be chosen the probability of taking one of them is $1 / g_{j k}$. The probability that a particular node $i$ lies on the geodesics between $j$ and $k$ is denoted by $g_{j i k}$. The betweenness $B$ for node $i$ is defined as the sum of these probabilities over all pairs of nodes not including node $i . B(i)=M \sum_{j, k} g_{j i k} / g_{j k}$, $M$ being some normalization constant. $B$ has a minimum value of zero when $i$ falls on no geodesics and a maximum at $(N-1)(N-2) / 2$, which is the number of pairs of nodes not including $i$. We use a relative version, i.e. $M$ is such that the sum of $B(i)$ over all $N$ nodes adds up to $100 \%$. Finally, the average path length in the (undirected) interbank connection network $A$ is $\bar{\ell}(A)=2.26 \pm 0.03$. $A$ is unweighted, meaning $A_{i j}=1$ for $A_{i j}^{w} \neq=0$ and $A_{i j}=0$ else. From these results the Austrian interbank network looks like a very small world with about three degrees of separation. This result looks natural in the light of the community structure described earlier. The two and three tier organization with head institutions and sub-institutions apparently leads to short interbank distances via the upper tier of the banking system and thus to a low degree of separation.

The framework here is a model of a banking system based on a detailed description of the structure of interbank exposures $L$ [7. The model explains the feasible payment flows between banks endogenously from the given structure of interbank liabilities, net values of the banks arising from all other bank activities and an assumption about the resolution of insolvency for different random draws from a distribution of risk-factor changes, such as interest rate, foreign exchange rate and stockmarket changes, as well as changes in default frequencies for corporate loans. We expose the banks' financial positions apart from interbank relations to interest rate, exchange rate, stock market and business cycle shocks. For each state of the world, the network model uniquely determines endogenously actual, feasible interbank payment flows. Taking the feedback between banks from mutual credit exposures and mutual exposures to aggregate shocks explicitly into account we can calculate default frequencies of individual banks across states. The endogenously determined vector of feasible payments between banks also determines the recovery rates of banks with exposures to an insolvent counterparty. We are able to distinguish bank defaults that arise directly as a consequence of movements in the risk factors and defaults which arise indirectly because of contagion. The model therefore yields a decomposition into fundamental and contagious defaults. Risk scenarios are created by exposing those positions on the bank balance sheet that are not part of the interbank business to interest rate, exchange rate, stock market and loan loss shocks. In order to do so we undertake a historic simulation using market data, except for the loan losses where we employ a credit risk model. In the scenario part we use data from Datastream, the major loans register, as well as statistics of insolvency rates in various industry branches from the Austrian rating agency (Kreditschutzverband von 1870). For each scenario the estimated matrix of bilateral exposures $L$ and the income positions determine via the network model a unique vector of feasible interbank payments and thus a pattern of insolvency. The basic idea is to determine the feasible flows of funds in the banking network that can occur 


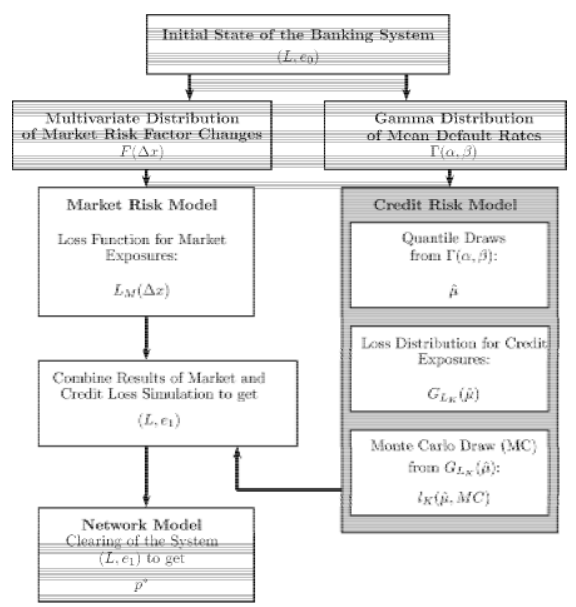

Fig. 2. Schematic diagram of the default flow model: At the initial state each bank is fully characterized by its wealth $e_{0}$ and its liabilities to other banks $L$. We expose each bank to stochastic shocks which model the market risk and the credit risk separately. The introduced risk changes the positions of the banks to new levels $e_{1}$. The crucial step is the clearing of the system where the structure of $L$ becomes important. The result of the clearing is a vector $p_{i}^{*}$ of payments bank $i$ has to pay to the other banks in the system. If a component of this vector becomes less than the obligations $d_{i}$ to pay to all the other banks $d_{i}=\sum_{j} L_{i j}$ bank $i$ is insolvent.

after a realization of risk factor changes by a clearing procedure in which creditors that can not fulfill their payment promises $(L)$ are proportionally rationed. One can show that a unique clearing vector always exists under mild regularity conditions 12. The clearing vector can be found constructively as follows: for the given portfolio positions of banks $e_{i}$ and the given risk factor changes we assume that every bank would pay its interbank liabilities as specified by $L$. If under this assumption one bank has a negative net value it is insolvent and its creditors receive a proportional claim on the remaining value of the insolvent bank. All banks with positive value are assumed to honor their promises fully. In a second iteration it can happen that under the new vector of feasible payment flows banks that were previously solvent are now insolvent because the value of their interbank claims is reduced impairing their capacity to pay. One can show that this procedure converges to a unique clearing payment vector $p^{*}$ for the system as a whole. From the clearing vector found in this way one can directly read three pieces of information: first all banks that have a component in the vector that is smaller than the sum of their promises as specified by $L$ are insolvent. The loss given default can be determined because it requires only a comparison between what has been promised (given by $L$ ) and what value can actually be payed (given by the clearing payment vector). Furthermore, insolvency cases can be distinguished by their occurrence in the clearing algorithm. Banks that are insolvent in the first round are fundamentally insolvent, others are insolvent because the value of interbank claims has been reduced by the insolvency of others. Their insolvency can therefore be interpreted as contagious. The analysis of 
Table 1. Simulation results for probabilities of fundamental and contagious defaults. A fundamental default is due to losses arising from exposures to market and non-bank credit risk. Contagious defaults are triggered by the default of another bank that cannot fulfill its promises. The probability that only fundamental defaults occur is shown as well as the probability that fundamental and contagious defaults are observed.

\begin{tabular}{|l|rrr|}
\hline Fundamental Defaults & No Contagion & Contagion & Total \\
\hline $0-10$ & $93.38 \%$ & $0.01 \%$ & $93.39 \%$ \\
11 to 20 & $2.82 \%$ & $0.40 \%$ & $3.22 \%$ \\
21 to 30 & $0.11 \%$ & $1.04 \%$ & $1.15 \%$ \\
31 to 40 & $0.00 \%$ & $0.40 \%$ & $0.40 \%$ \\
41 to 50 & $0.00 \%$ & $0.53 \%$ & $0.53 \%$ \\
Total & $96.31 \%$ & $3.69 \%$ & $100.00 \%$ \\
\hline
\end{tabular}

these data then allows us to assess the risk exposure - in particular for defaults - of all banks at a system level. The details of the model are described in [7].

\section{Results and Conclusions}

The given banking system is very stable and default events that could be classified as a "systemic crisis" are unlikely. We find that the mean default probability of an Austrian bank to be below one percent. Perhaps the most interesting finding is that only a small fraction of bank defaults can be interpreted as contagious. The vast majority of defaults is a direct consequence of macroeconomic shocks. More specifically, we find the median endogenous recovery rates to be $66 \%$, and we show that the given banking system is quite stable to shocks from losses in foreign counterparty exposure and we find no clear evidence that the interbank market either increases correlations among banks or enables banks to diversify risk. Using our model as a simulation tool, we show that market share in the interbank market alone is not a good predictor of the relevance of a bank for the banking system in terms of contagion risk. Table 1 shows the decomposition of simulation scenarios with and without contagion following a fundamental default of a given number of banks. The simulation is run under the assumption that there is a recovery rate of $50 \%$ of loans to non banks and that the full value of an insolvent institution is transferred to the creditors. Clearing is done after the netting of interbank claims in $L$.

Finally, we ask the question of the impact of individual bank defaults on other banks. More specifically, if one particular bank becomes erased from the network, how many other banks become insolvent due to this event? We call this conditional contagion impact on default of a specific bank. This is similar in spirit to looking at avalanche distributions triggered by controlled toggling of grains in a sandpile. We observe the portfolio positions $e_{i}$ of banks from our database. Instead of simulating risk factor changes that are applied to this portfolio and are then cleared in a second step we artificially eliminate the funds of each bank one at a time, clear the system, and count the number of contagious (induced) defaults. This is repeated $N$ times so that each bank becomes removed, and all 
the others are present. We find that only 13 banks - when defaulting - drag more than one other bank into default. There are 16 banks which will cause one single default of one other bank. A natural guess would be to relate the contagion impact of a specific bank to its role in the network. Amongst many possible measures, we find that the betweenness of the defaulting bank is directly related to the contagion impact. This is shown in Fig. 1 c, where a linear relation between the betweenness $B(i)$ and the contagion impact is suggested for $B(i)>2$.

In this work we combined the knowledge of the detailed structure of a real world banking network with an economic model, which allows to estimate the functional stability and robustness of the financial network. This model adds a dynamical component to the "static" liability matrix, which is nothing else but the promise for future financial flows between the banks. By stochastically varying external parameters of the system like interest rate shocks, we can follow the flow of financial transactions, and in particular can scan for defaults occurring in the system, due to insolvency. The results of this work is that the system seems to be relatively robust, and the probability for a contagious spread over the whole network is very small. However, there are several key banks (not including the Central bank), which upon their default, will lead to a considerable number of other banks defaulting as well. We showed that these key banks can be reliably identified by the vertex betweenness. We think that the existence of a threshold in the variable $B(i)$ in a "quasi" scale-free network, combined with complex but realistic contagion dynamics is a remarkable finding which is worthwhile to examine further.

\section{References}

1. Dorogovtsev, S.N. and Mendes, J.F.F.: Evolution of Networks: From Biological Nets to the Internet and WWW, Oxford University Press (2003)

2. Hartmann, P. and DeBandt, O.: Systemic Risk: An Overview. European Central Bank Working Paper (2000) 35

3. Summer, M.: Banking Regulation and Systemic Risk. Open Econ. Rev. 1 (2003) 43

4. Allen, F. and Gale, D.: Financial Contagion. J Polit. Econ. 108 (2000) 1

5. Thurner, S., Hanel, R., Pichler, S.: Risk trading, network topology, and banking regulation. Quantitative Finance 3 (2003) 306

6. Boss, M., Elsinger, H., Summer, M., Thurner, S.: The Network Topology of the Interbank Market. SFI working paper \# 03-10-055; cond-mat/0309582 (2003)

7. Elsinger, H., Lehar, A., Summer, M.: Risk Assessment of Banking Systems. Austrian National Bank working paper 79 (2002).

8. Tadić, B., Thurner, S.: Information Super-Diffusion on Structured Networks, Physica A 332 (2004) 662

9. Tadić, B., Thurner, S., Rodgers, G. J.: Traffic on complex networks: Towards understanding global statistical properties from microscopic density fluctuations. Phys. Rev. E (2004) in press

10. Zhou, H.: Distance, dissimilarity index, and network community structure. Phys. Rev. E 67 (2003) 061901

11. Girvan, M. and Newman, M.E.J.: Proc. Natl. Acad. Sci. 99 (2002) 7831

12. L. Eisenberg and T. Noe, Systemic Risk in Financial Systems. Management Science 47 (2001) 236 